\title{
Genomic and Molecular Mechanisms for Efficient Biodegradation of Aromatic Dye in Presence of Lignin
}

\author{
Su Sun, Hongbo Yu, Xiaoyu Zhang, Shangxian Xie \\ Department of Biotechnology, College of Life Science and Technology, Huazhong University of Science and Technology \\ 1037 Luoyu Road, Wuhan, 43004, China \\ sunsu@hust.edu.cn; yuhongbo@hust.edu.cn; zhangxiaoyu@hust.edu.cn; shangxian_xie@hust.edu.cn
}

\section{Extended Abstract}

Understanding the genomic and molecular mechanisms for aromatic compound degradation is crucial for the development of effective bioremediation strategies. We hereby report the discovery of a novel phenomenon for improved Direct Red 5B (DR5B) azo dye degradation by Irpex lacteus CD2 with lignin as a co-substrate. This promoted phenomenon provides an ideal system for studying the mechanisms of polycyclic aromatic dye and lignin degradation. Multiple integrated "omics" was carried out to elucidate the molecular mechanisms for aromatic degradation in white rot fungus by comparing dye, lignin, and dye/lignin combined treatments. The gene category analysis revealed many aromatic polymer degradationrelevant pathways were coordinately up-regulated under the combined treatment. Firstly, a full spectrum of lignin degradation peroxidases, oxidases, radical producing enzymes and other relevant components, such as metal binding and ion transporter related proteins were up-regulated under DR5B and lignin treatments. Lignin induced genes well complement the DR5B induced genes to provide essential enzymes and redox conditions for aromatic compound degradation. Secondly, besides the lignolytic enzymes, Fenton reaction based on hydroxyl radical and iron might also play a critical role in DR5B and lignin degradation. Meanwhile, Manganese perioxidase (MnP) was found to be the key enzyme required for the rapid degradation of DR5B degradation by I. lacteus CD2, which was further confirmed by in vitro dye degradation assay with purified MnP. The "omics" analysis also showed that the aromatic compound catabolism related enzymes including homogentisate 1,2-dioxygenase were significantly overexpressed in co-substrate system which was believed to be the key enzymes for the degradation pathway in I. lacteus CD2. The result showed that effective aromatic polymer degradation requests the synergistic enzymes and radical-mediated oxidative reactions to form an effective network of chemical processes. Overall, this study established an effective dyes biodegradation system with lignin as co-substrate by white rot fungi and revealed the molecular mechanisms and dynamic degradation process for effective aromatic compounds degradation, which will help to guide the development of effective bioremediation and biomass degradation strategies. 